\title{
Modelo de gestión de servicios ITIL para E-learning
}

\author{
José Ignacio Palacios-Osma, José Luis Rodríguez-Guzmán \& Claudia Ximena García-Ramírez \\ Facultad de Ingeniería, Universidad Distrital Francisco José de Caldas, Bogotá, Colombia.jpalacios@udistrital.edu.co, \\ joslrodriguezg@correo.udistrital.edu.co,cxgarciar@correo.udistrital.edu.co
}

\begin{abstract}
Resumen - Este artículo presenta un modelo de gestión para un sistema de educación con metodología virtual, generado a partir del estudio realizado sobre un programa de educación con metodología virtual de la Universidad Distrital Francisco José de Caldas en Bogotá, Colombia. Para la construcción del modelo se realiza una evaluación de los procesos propuestos por la Librería de Infraestructura de Tecnologías de Información (ITIL) frente a 4 componentes propios de un sistema de educación virtual: tecnológico, administrativo, comunicativo y pedagógico, se define el alcance y las responsabilidades de los actores involucrados en el funcionamiento del sistema, y se plantea un listado de actividades a desarrollar para cada etapa del ciclo de vida del servicio.
\end{abstract}

Palabras Clave-ITIL, Modelo de gestión, E-Learning, Educación virtual, Procesos.

Recibido: 13 de abril de 2016. Revisado: 14 de septiembre de 2016. Aceptado: 20 de septiembre de 2016.

\section{ITIL services management model for E-learning}

Abstract- This paper presents a management model for a virtual methodology education system generated from a Universidad Distrital Francisco Jose de Caldas virtual education program research in Bogota, Colombia. For the model construction we evaluate the proposal process of the Information Technology Infrastructure Library (ITIL) with four virtual education components, technological, administrative, communicative and educational, then the scope and responsibilities of the actors involved in the operation of the system was define, and a list of activities was propose to be developed for each stage of the service lifecycle. process.

Keywords - ITIL, management model, E-Learning, virtual education,

\section{Introducción}

La educación virtual hoy en día presenta una serie de desafíos que deben ser resueltos para garantizar su calidad; necesita estándares y buenas prácticas que faciliten y mejoren sustancialmente las condiciones bajo las cuales funciona. Por ésta razón ITIL, que proporciona buenas prácticas que cubren las actividades más importantes de una organización de servicios basada en Tecnologías de Información (TI) [1] se convierte en una excelente alternativa para la gestión de un sistema de educación virtual.

El presente artículo evidencia el uso de las buenas prácticas de ITIL en los procesos de educación virtual y plantea un modelo que apoya la gestión, desarrollo y el correcto funcionamiento de éste servicio tanto en provisión como en soporte, generando una metodología que pueda guiar el proceso de implementación de cualquier programa académico con metodología virtual y en donde se evidencian relaciones entre los distintos procesos involucrados en la prestación del servicio. Para lograrlo se realizó una búsqueda bibliográfica de información referente tanto a educación virtual, su evolución y sus necesidades, como de casos de aplicación de buenas prácticas ITIL en el mundo, identificando objetivos, metodologías y resultados, que sirvieron como guía para el desarrollo de esta investigación, además se realizó un trabajo de campo en la Universidad Distrital, en donde se encontró el acompañamiento y apoyo de la coordinación de la Maestría en Telecomunicaciones móviles en metodología virtual para la consecución de nuestro objetivo.

\section{Marco teórico}

\subsection{Educación virtual}

El modelo de enseñanza tradicional conformado por clases magistrales, en donde el alumno realiza toma apuntes, desarrolla habilidades relativas a la búsqueda de información en distintas fuentes y afronta un proceso de evaluación continua[2] ha caracterizado la educación desde el siglo pasado. Sin embargo necesidades como: educación a lo largo de las distintas etapas de la vida, desaparecer las barreras espacio-temporales y tener horarios académicos flexibles[3] dan pie a una metodología de educación distinta que nace como resultado de la implementación de tecnologías de la información y la comunicación (TIC) y la creación de sistemas de acceso a la red como un modelo de enseñanza y aprendizaje[4] denominado educación virtual.

La educación virtual posee características particulares en el proceso de enseñanza como la independencia y autonomía[4] ya que son los estudiantes quienes gestionan y disponen de su ritmo de trabajo; además posee herramientas de Tecnologías de la Información (TI) para lograr que la experiencia de los usuarios sea interactiva y dinámica, desplazando el punto focal del docente al estudiante y de la enseñanza al aprendizaje[5]. El entorno de la virtualidad se conforma por cuatro componentes

Como citar este artículo: Palacios-Osma, J. I., Rodríguez-Guzmán, J. L. \& García-Ramírez, C. X.. Modelo de gestión de servicios ITIL para e-learning

. Revista Educación en Ingeniería 12 (23) 28-34, Febrero, 2017. 
identificados como: pedagógico, tecnológico, administrativo y comunicativo[6], los cuales demandan a su vez diferentes niveles de servicio y de buenas prácticas.

\subsection{ITIL}

La biblioteca de infraestructura de tecnologías de información (ITIL) nace por la necesidad de incorporar TI en las organizaciones para alcanzar sus objetivos corporativos, puesto que en un entorno globalizado es indispensable contar con servicios de TI con altos estándares de calidad que soporten el cumplimiento de dichos objetivos.

ITIL es un conjunto de conceptos y prácticas que permiten la gestión del servicio de TI, su desarrollo y el de las operaciones que intervienen en ésta[7]. Para éste fin ITIL describe de manera minuciosa los procedimientos de gestión diseñados para alcanzar niveles de calidad y productividad óptimos en prestación de servicios TI.

La librería ha experimentado una transformación desde su primera versión y en la actualidad se ha constituido como un estándar en cuanto a gestión de servicios de TI[8]. En su última versión describe el ciclo de vida de servicio, como se evidencia en la Fig. 1.

Distintos trabajos de investigación han demostrado notables mejoras en la gestión de servicios TI. La investigación realizada en Hangzhou, China[10] en la cual se implementaron procesos ITIL en una empresa que fabrica prendas de vestir, demuestra una reducción de costos asociados a la solución de problemas e incidentes, debido a los cortos tiempos de respuesta y la adecuada gestión de recursos y conocimiento que propició su aplicación. Latrache, Nfaoui, \& Boumhidi,[11] proponen un algoritmo basado en un modelo multi-agente a través del cual busca automatizar el proceso de respuesta a incidentes, para lo cual plantea un listado de actividades a desarrollar en su algoritmo a partir de los lineamientos del proceso de gestión de incidentes de ITIL, y obtiene como resultado una reducción en recursos y tiempos de respuesta a incidentes. Zhang, Wang, Peng ; Zhigang \& Zong [12], integran los procesos de ITIL con las técnicas organizacionales de una compañía dedicada a la prestación de servicios web, y evidencia que éstos procesos propios de ITIL no solo se integran con la organización, sino también a través de las fronteras organizativas que facilitan las

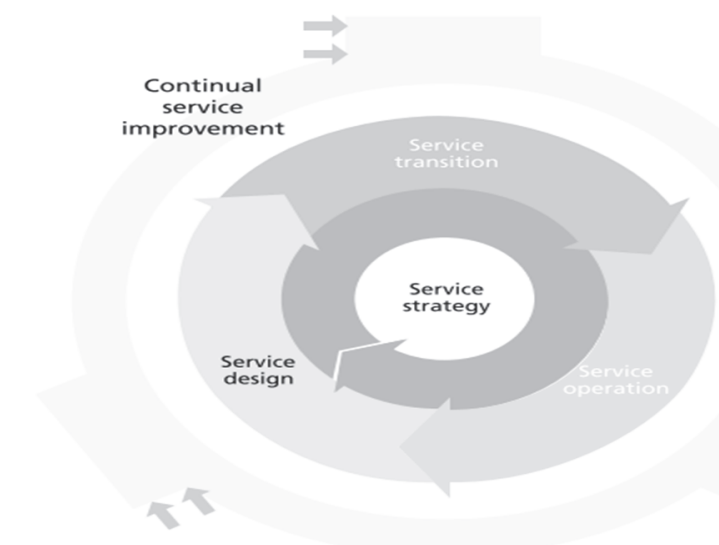

Figura 1 Ciclo de Vida del Servicio ITIL V3. Fuente: Service Strategy ITIL [9]. operaciones de colaboración y de intercambio de conocimientos en internet.

Aunque la cantidad de investigaciones realizadas basadas en implementación de las buenas prácticas ITIL es numerosa, no es fácil hallar trabajos que aborden la temática de educación virtual; sin embargo para el caso de estudio se escogieron 3 trabajos en donde se implementa ITIL en educación superior en busca del mejoramiento de sus procesos.

Tituaña Davila \& Yacchirema Vargas[13] elaboran un modelo de gestión denominado e-SUE el cual integra las buenas prácticas de ITIL en la búsqueda del mejoramiento continuo de la calidad del servicio del sistema Universitario Ecuatoriano bajo la modalidad de E-learning, esto a través de una metodología en donde se definen roles y actividades para el correcto funcionamiento del sistema de educación.

Lagua[14]desarrolla reingeniería de procesos utilizando ITIL en la Dirección de Tecnología de Información y Comunicación (DITIC) de la Universidad Técnica de Ambato, Ecuador, para el mejoramiento de la estrategia, diseño y gestión de problemas en búsqueda de minimizar los incidentes en esta Dirección encargada de brindar servicios tecnológicos a toda la comunidad universitaria.

Conza \& Medrano[15] realizaron un análisis de riesgos a partir del cual elaboraron el Plan de Continuidad de la Unidad de Educación Virtual de la Escuela Politécnica Nacional de Quito-Ecuador, utilizando el proceso Gestión de Continuidad de la librería ITIL para la asignación de recursos e implementación de su metodología.

\section{Metodología}

La construcción del modelo de gestión de servicio de educación con metodología virtual, basado en prácticas ITIL, se desarrolló de la siguiente manera:

1. Se estudiaron los procesos de la Maestría en Telecomunicaciones móviles de la Universidad Distrital.

2. Se determinaron los procesos ITIL que contribuyen al desarrollo y la mejora del programa virtual respecto a cada componente estudiado.

3. Se determinó la importancia de cada componente frente a los procesos ITIL para los programas con metodología virtual.

4. Se elaboró un modelo basado en prácticas ITIL que soporta e interrelaciona los 4 componentes de la educación virtual.

Dado el alcance de la propuesta, se abordaron 16 de los 30 procesos del ciclo de vida del servicio, para ello se realizó un examen basado en dos criterios, Urgencia y viabilidad [10], dando así prioridad a los procesos que se enuncian a continuación: Gestión de Portafolio de servicios, Gestión Financiera, Gestión de la Demanda, Gestión de Relaciones de negocios, Gestión del Catálogo de servicios, Gestión de Niveles de servicio, Gestión de Capacidad, Gestión de Disponibilidad y Gestión de Seguridad del servicio (debido a que es un programa nuevo que busca fortalecerse) y los procesos de Gestión del Conocimiento, Gestión de Eventos, Gestión de Incidentes, Gestión de Problemas, Gestión de Acceso a los servicios y Cumplimiento de requerimientos (debido a que es un servicio que debe estar disponible y en operación 24 horas 7 días a la 
Tabla 1

Resumen de interacciones: Procesos ITIL - Componentes educación virtual

\begin{tabular}{|c|c|c|c|c|}
\hline & $\begin{array}{l}\text { Pedagó } \\
\text { gico }\end{array}$ & $\begin{array}{l}\text { Comunic } \\
\text { ativo }\end{array}$ & $\begin{array}{l}\text { Tecnoló } \\
\text { gico }\end{array}$ & $\begin{array}{l}\text { Administ } \\
\text { rativo }\end{array}$ \\
\hline $\begin{array}{l}\text { Gestión de portafolio } \\
\text { de servicios }\end{array}$ & $\mathrm{X}$ & $\mathrm{X}$ & $\mathrm{X}$ & $\mathrm{X}$ \\
\hline $\begin{array}{l}\text { Gestión Financiera } \\
\text { para servicios TI }\end{array}$ & & & & $\mathrm{X}$ \\
\hline Gestión de la Demanda & & & $\mathrm{X}$ & \\
\hline $\begin{array}{l}\text { Gestión de relaciones } \\
\text { de negocios }\end{array}$ & & $\mathrm{X}$ & $\mathrm{X}$ & $\mathrm{X}$ \\
\hline $\begin{array}{l}\text { Gestión del catálogo de } \\
\text { servicios }\end{array}$ & $\mathrm{X}$ & $\mathrm{X}$ & $\mathrm{X}$ & \\
\hline $\begin{array}{l}\text { Gestión de los niveles } \\
\text { de servicio }\end{array}$ & $X$ & $X$ & $\mathrm{X}$ & $\mathrm{X}$ \\
\hline Gestión de la capacidad & & & $\mathrm{X}$ & \\
\hline $\begin{array}{l}\text { Gestión de la } \\
\text { disponibilidad }\end{array}$ & & & $\mathrm{X}$ & \\
\hline $\begin{array}{l}\text { Gestión de la seguridad } \\
\text { del servicio }\end{array}$ & & $\mathrm{X}$ & $\mathrm{X}$ & $\mathrm{X}$ \\
\hline $\begin{array}{l}\text { Gestión del } \\
\text { conocimiento }\end{array}$ & & & $\mathrm{X}$ & $\mathrm{X}$ \\
\hline Gestión de eventos & & & $\mathrm{X}$ & \\
\hline Gestión de Incidentes & & & $\mathrm{X}$ & \\
\hline $\begin{array}{l}\text { Cumplimiento de } \\
\text { requerimientos }\end{array}$ & & & $\mathrm{X}$ & \\
\hline Gestión de problemas & & & $\mathrm{X}$ & \\
\hline $\begin{array}{l}\text { Gestión de acceso a los } \\
\text { servicios }\end{array}$ & $X$ & $\mathrm{X}$ & $\mathrm{X}$ & $\mathrm{X}$ \\
\hline Sumatoria & 4 & 6 & 14 & 7 \\
\hline Porcentaje & $13 \%$ & $19 \%$ & $\begin{array}{l}45 \\
\%\end{array}$ & $23 \%$ \\
\hline
\end{tabular}

Fuente: Elaboración Propia.

semana), además se incluyó la etapa de mejora continua, la cual es transversal a todas las etapas del ciclo de vida del servicio y es la encargada de evaluar y hacer seguimiento a todos los procesos de la organización, recopilando información propia de cada incidente que permite diseñar e implementar contingencias y proponer mejoras en los demás procesos.

Una vez definidos los procesos fundamentales ITIL para el caso de estudio, se analizó su relación con cada componente de la educación virtual y se discriminó con una $\mathrm{x}$ las relaciones existentes como se muestra en la Tabla 1.

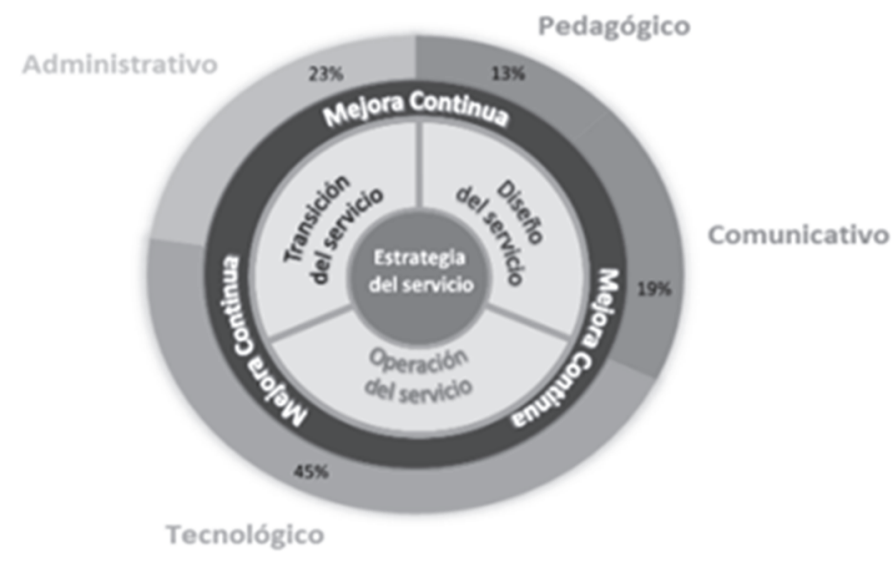

Figura 2. Ciclo de vida ITIL en relación a los componentes de la Educación virtual.

Fuente: Elaboración propia
Después se realizó un conteo de las relaciones por componente y se calculó el peso de cada uno frente a los procesos ITIL asignando un porcentaje respecto al total de relaciones encontradas. A partir de esto se diseñó la Fig. 2, que muestra que el $45 \%$ del ciclo de vida del servicio de educación con metodología virtual está soportado por el componente tecnológico, conformado en su mayoría por los procesos de operación y transición del servicio, mientras los componentes administrativo pedagógico y comunicativo $(23 \%, 13 \%$ y $19 \%$ respectivamente), se fundamentan en los procesos de estrategia, diseño y transición del servicio.

\section{Resultados y Análisis}

\subsection{Modelo de gestión para la educación virtual (MGEV)}

Para la elaboración del modelo de gestión MGEV se relacionaron los procesos ITIL con los actores que intervienen en la prestación del servicio y de quienes depende su correcto funcionamiento.

El modelo que aparece en la Fig. 3 recopila y estructura los procesos a lo largo del ciclo de vida ITIL, como herramienta para la gestión del servicio de educación virtual, aplicado para el caso de estudio en la Maestría en Telecomunicaciones móviles de la Universidad Distrital, en donde las responsabilidades asociadas a cada uno de los actores que intervienen son:

\subsubsection{Ministerio de Educación de Colombia}

Responsable de exigir a las entidades de educación contenidos, recursos y ambientes de aprendizaje acordes con unos estándares de calidad preestablecidos.

\subsubsection{Universidad}

Responsable de brindar un servicio de calidad, que responda a las necesidades de los estudiantes y a los requerimientos del Ministerio de Educación.

\subsubsection{Estudiantes}

Responsables de exigir contenidos académicos de calidad y recursos adecuados para sus actividades de aprendizaje, identificar inconformidades y realizar sugerencias, quejas y peticiones, en primera instancia a la Universidad y en segunda al Ministerio de Educación.

A partir del estudio de los lineamientos institucionales descritos en el Plan Estratégico de Incorporación de Medios y Tecnologías de la Información en los procesos educativos de la Universidad Distrital [16] y las necesidades encontradas a partir de la investigación se establecieron actividades a desarrollar durante cada proceso de cada una de las etapas del ciclo de vida que buscan satisfacer las necesidades propias de cada componente de educación virtual como se muestra en las Tablas $2-5$. 


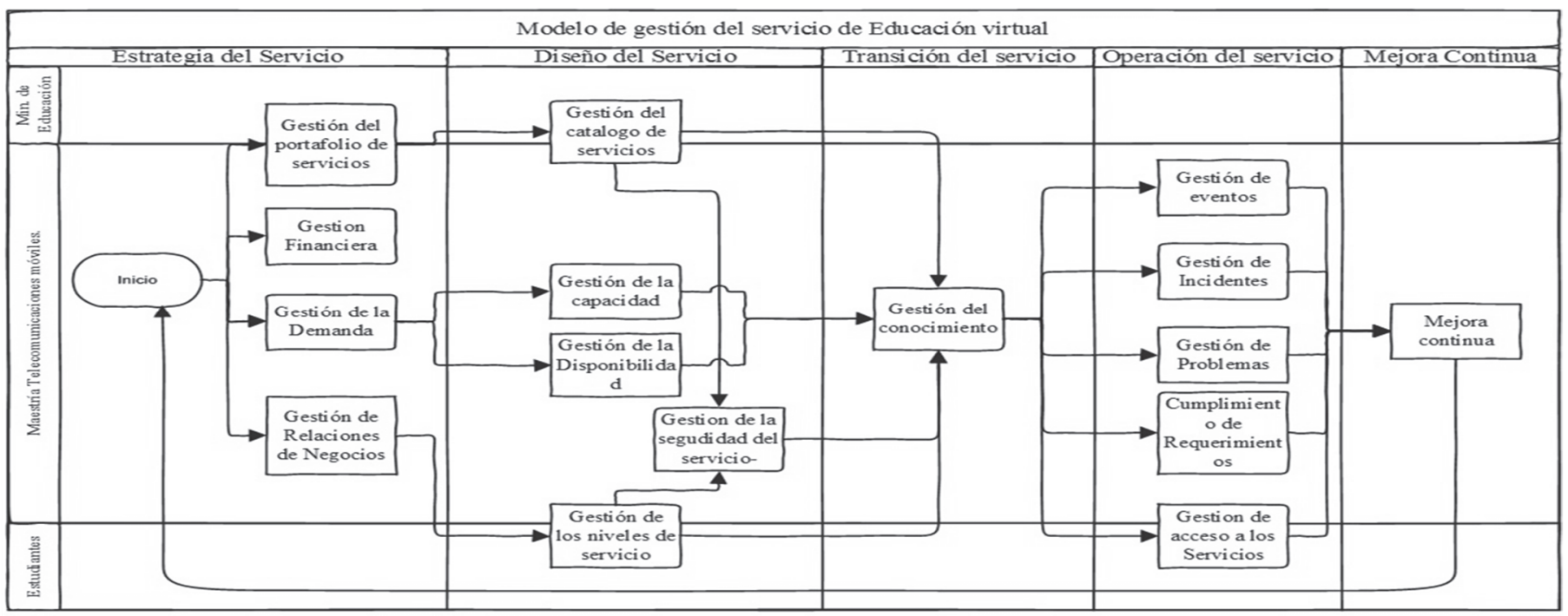

Figura 3. Modelo de Gestión MGEV

Fuente: Elaboración Propia.

En la Tabla 2 están enumeradas las actividades planteadas para la etapa de estrategia del servicio, quien debe brindar lineamientos para establecer y dar prioridad a los objetivos del programa virtual, para ello se debe estudiar su mercado y armonizar la relación de oferta/demanda para proponer

Tabla 2

Actividades a desarrollar durante Estrategia del Servicio, Proceso ITIL.

\begin{tabular}{|c|c|c|}
\hline $\begin{array}{l}\text { Estrategia del } \\
\text { servicio }\end{array}$ & & Actividades \\
\hline $\begin{array}{l}\text { Gestión } \\
\text { Financiera }\end{array}$ & 1 & $\begin{array}{l}\text { Consolidar estrategias para el crecimiento y } \\
\text { adecuación de espacios virtuales que garanticen } \\
\text { herramientas que generen ambientes de } \\
\text { aprendizaje donde el alumno, el tutor y la } \\
\text { universidad interactúan y construyen un programa } \\
\text { académico de calidad. }\end{array}$ \\
\hline & 2 & $\begin{array}{l}\text { Gestionar la adquisición de equipos y plataformas } \\
\text { permitiendo viabilidad y competitividad de los } \\
\text { programas de educación virtual. }\end{array}$ \\
\hline Gestión & 3 & $\begin{array}{l}\text { Desarrollar contenidos que involucren las } \\
\text { actividades de aprendizaje del alumno y las } \\
\text { herramientas colaborativas que faciliten la } \\
\text { apropiación del conocimiento. }\end{array}$ \\
\hline $\begin{array}{l}\text { Portafolio de } \\
\text { servicios }\end{array}$ & 4 & $\begin{array}{l}\text { Establecer contenidos multimedia y herramientas } \\
\text { de interactividad que permitan dinamismo en la } \\
\text { plataforma. }\end{array}$ \\
\hline & 5 & $\begin{array}{l}\text { Definir la infraestructura TIC que soporte la } \\
\text { construcción de procesos enseñanza-aprendizaje y } \\
\text { cumplimiento de los estándares establecidos. }\end{array}$ \\
\hline $\begin{array}{l}\text { Gestión } \\
\text { Demanda }\end{array}$ & 6 & $\begin{array}{l}\text { Soportar mediante la infraestructura de servidores } \\
\text { los servicios concurrentes bajo demanda y } \\
\text { asegurar la prestación, alojamiento y } \\
\text { almacenamiento en el despliegue de los servicios. }\end{array}$ \\
\hline \multirow{3}{*}{$\begin{array}{l}\text { Gestión } \\
\text { Relaciones de } \\
\text { negocios }\end{array}$} & 7 & $\begin{array}{l}\text { Establecer relaciones que se generan en el } \\
\text { intercambio comunicativo alumno-docente } \\
\text { alumno-alumno. }\end{array}$ \\
\hline & 8 & $\begin{array}{l}\text { Establecer relaciones causales que se generan entre } \\
\text { docente-estudiante con los recursos asignados a } \\
\text { cada uno de ellos. }\end{array}$ \\
\hline & 9 & $\begin{array}{l}\text { Establecer relaciones entre los actores que } \\
\text { intervienen en el proceso de planeación, diseño y } \\
\text { operación del servicio garantizando la integración } \\
\text { de conocimientos. }\end{array}$ \\
\hline
\end{tabular}

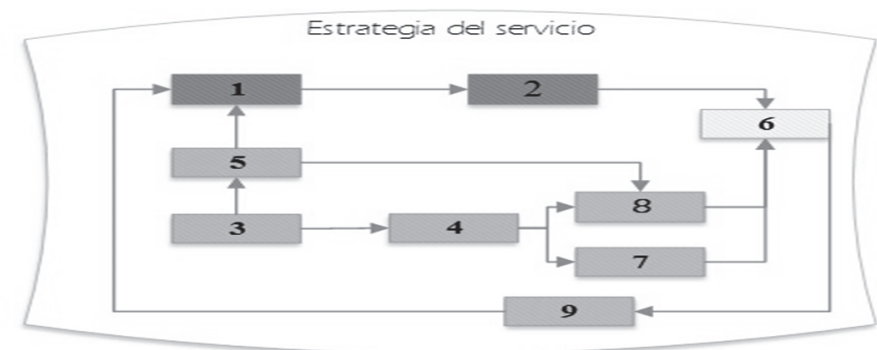

Figura 4. Diagrama de Flujo Estrategia del Servicio. Fuente: Elaboración Propia

servicios que aporten valor añadido. Es indispensable gestionar los recursos necesarios para prestar los servicios que se ofrecen teniendo en cuenta sus costos y riesgos asociados. Lo más importante durante esta etapa es elaborar planes que permitan un crecimiento sostenible, motivo por el cual se convierte en una etapa de retroalimentación que guía y soporta el programa académico.

Cada actividad de esta etapa se relaciona de manera directa con una o más de ellas, por lo cual se requiere sinergia en la ejecución del conjunto para garantizar un correcto desempeño del modelo planteado, la Fig. 4 evidencia dichas relaciones e invitan a seguir de forma lógica cada una de ellas.

Diseño del servicio es una etapa que depende estrictamente de los objetivos y lineamientos impartidos por la etapa anterior, debe encargarse de adecuar los servicios prestados por el programa académico a las necesidades del mercado y funciona como un evaluador de capacidad, seguridad, disponibilidad y niveles de servicio.

Durante esta etapa se identifican requerimientos que deben ser satisfechos, algo indispensable para la retroalimentación que necesita el ciclo de vida del servicio ITIL, Las actividades establecidas para el Diseño del servicio se muestran en la Tabla 3. 
Tabla 3

Actividades a desarrollar durante Diseño del Servicio, Proceso ITIL

\begin{tabular}{|c|c|c|}
\hline $\begin{array}{c}\text { Diseño del } \\
\text { servicio }\end{array}$ & & Actividades \\
\hline \multirow{4}{*}{$\begin{array}{l}\text { Gestión Catálogo } \\
\text { de servicios }\end{array}$} & 10 & $\begin{array}{l}\text { Revisar y ajustar el portafolio de servicios si resulta } \\
\text { pertinente, de acuerdo a las necesidades del estudiante } \\
\text { con base en los modelos pedagógicos utilizados en el } \\
\text { aprendizaje. }\end{array}$ \\
\hline & 11 & $\begin{array}{l}\text { Implementar contenidos digitales de forma idónea } \\
\text { para que sea recibido de forma eficiente en el proceso } \\
\text { de aprendizaje. }\end{array}$ \\
\hline & 12 & $\begin{array}{l}\text { Proponer servicios de modernización y mejoramiento } \\
\text { de procesos. }\end{array}$ \\
\hline & 13 & $\begin{array}{l}\text { Actualizar permanentemente contenidos estipulados } \\
\text { en el plan de desarrollo del curso virtual } \\
\text { Establecer herramientas comunicativas entre alumno- }\end{array}$ \\
\hline \multirow{5}{*}{$\begin{array}{l}\text { Gestión Niveles } \\
\text { de servicio }\end{array}$} & 14 & $\begin{array}{l}\text { alumno y tutor-alumno, que permitan } \\
\text { retroalimentación en los procesos ejecutados. }\end{array}$ \\
\hline & 15 & $\begin{array}{l}\text { Diseño y actualización de protocolos en donde se } \\
\text { muestre calendario de actividades, horarios de } \\
\text { atención y los canales de comunicación que se } \\
\text { establecerán ya sean sincrónicos o asincrónicos. }\end{array}$ \\
\hline & 16 & $\begin{array}{l}\text { Asesorar el licenciamiento en software y la creación } \\
\text { y administración usuarios virtuales. }\end{array}$ \\
\hline & 17 & $\begin{array}{l}\text { Definir responsabilidades usuario-servicio en cuanto } \\
\text { a seguridad, información, infraestructura tecnológica, } \\
\text { de software y conectividad. }\end{array}$ \\
\hline & 18 & $\begin{array}{l}\text { Implementar políticas que generen un clima propicio } \\
\text { en la comunidad universitaria. }\end{array}$ \\
\hline \multirow[b]{2}{*}{$\begin{array}{l}\text { Gestión } \\
\text { Capacidad }\end{array}$} & 19 & $\begin{array}{l}\text { Garantizar el funcionamiento y la actualización del } \\
\text { campus virtual y la interoperabilidad SIUD }\end{array}$ \\
\hline & 20 & $\begin{array}{l}\text { Soporte CMS ( sistemas de } \\
\text { administración de contenido) y LMS (sistema de } \\
\text { gestión de aprendizaje ) }\end{array}$ \\
\hline \multirow{5}{*}{$\begin{array}{l}\text { Gestión } \\
\text { disponibilidad }\end{array}$} & 21 & Gestionar acceso a las plataformas $24 \times 7 \times 365$ \\
\hline & 22 & Establecer horario de Mesa de ayuda L-V 9-5. \\
\hline & 23 & $\begin{array}{l}\text { Asegurar confidencialidad en evaluaciones, y el } \\
\text { seguimiento en el progreso del curso. }\end{array}$ \\
\hline & 24 & $\begin{array}{l}\text { Definir mecanismos que aseguren que los módulos de } \\
\text { curso virtual correspondan con la temática indicada. }\end{array}$ \\
\hline & 25 & $\begin{array}{l}\text { Asegurar que el contenido cumpla con los estándares } \\
\text { establecidos de derechos de autor. }\end{array}$ \\
\hline \multirow{4}{*}{$\begin{array}{l}\text { Gestión } \\
\text { Seguridad del } \\
\text { servicio }\end{array}$} & 26 & $\begin{array}{l}\text { Proteger la información y regirse bajo normas de nivel } \\
\text { de acceso y confidencialidad. }\end{array}$ \\
\hline & 27 & Establecer planes y niveles de seguridad \\
\hline & 28 & Definir políticas de seguridad de acuerdo ISO27001 \\
\hline & 29 & $\begin{array}{l}\text { Monitorear el correcto acceso al contenido permitido. } \\
\text { Incluir en la metodología los aspectos legales que } \\
\text { intervienen directamente o tiene relaciones con la } \\
\text { maestría: estatuto estudiantes, docente, académico, } \\
\text { propiedad intelectual }\end{array}$ \\
\hline
\end{tabular}

Fuente: Elaboración Propia.

En el diagrama de flujo que se muestra en la Fig. 5. se evidencia la dependencia entre procesos que conforman esta etapa.

La etapa de transición de servicio debe gestionar toda la información relevante para la prestación del servicio con el fin de garantizar que esté disponible para los agentes implicados en el diseño, desarrollo, implementación y operación de cualquier actualización de los espacios virtuales, por lo tanto se vuelve el foco de MGEV; el punto en el cual convergen todas las actividades de gestión planteadas para este modelo, constituye el principal apoyo de la etapa de Mejoramiento continuo, la cual es transversal a todas las demás y requiere de toda la información acumulada para gestionar mejoras en los servicios prestados.

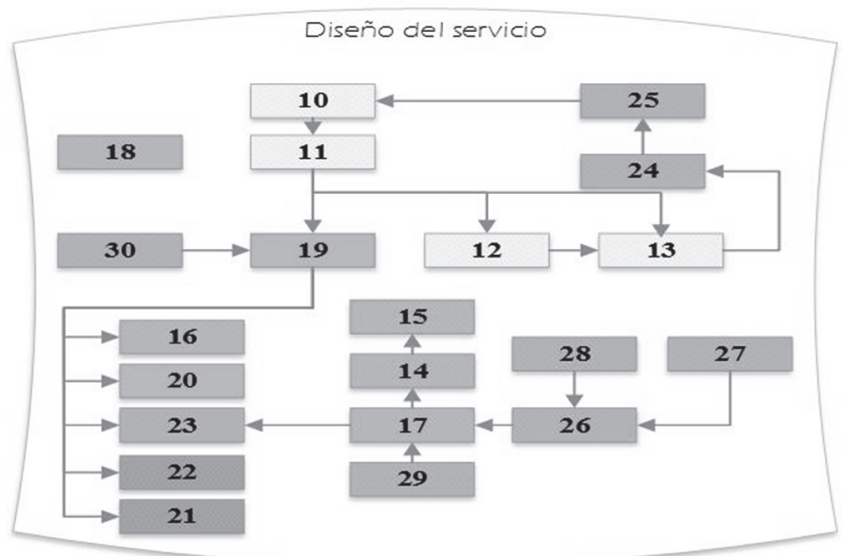

Figura 5. Diagrama de Flujo Diseño del Servicio.

Fuente: Elaboración Propia

Tabla 4

Actividades a desarrollar en Transición del Servicio, Proceso ITIL

\begin{tabular}{|c|c|c|}
\hline $\begin{array}{c}\text { Transición del } \\
\text { servicio }\end{array}$ & & Actividades \\
\hline \multirow[t]{2}{*}{$\begin{array}{l}\text { Gestión del } \\
\text { conocimiento }\end{array}$} & 31 & $\begin{array}{l}\text { Actualización de las plataformas, software y redes, } \\
\text { junto con un plan de implementación y despliegue } \\
\text { que garantice un proceso de cambios exitoso. }\end{array}$ \\
\hline & 32 & $\begin{array}{l}\text { Gestionar una base de datos de configuraciones, } \\
\text { donde se disponga de toda la información } \\
\text { relevante para su consulta y uso. }\end{array}$ \\
\hline
\end{tabular}

Fuente: Elaboración Propia.

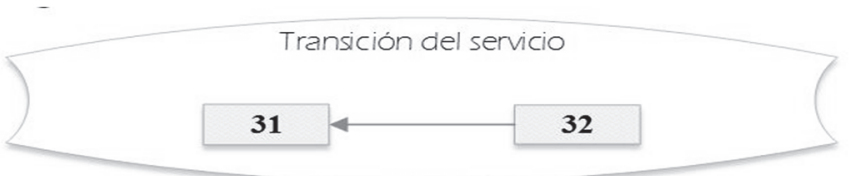

Figura 6. Diagrama de Flujo Transición del Servicio.

Fuente: Elaboración Propia

Aquí no solo se registran los incidentes que se presentan durante la operación y la forma en cómo deben solucionarse, sino que se registran todos los aspectos considerados como problemas en el servicio y se transforman en oportunidades de mejora. Para el proceso de Gestión de conocimiento propio de esta etapa se establecieron las actividades que aparecen en la Tabla 4.

El diagrama de flujo para esta etapa en este modelo inicial es sencillo, puesto que solo involucra dos actividades, sin embargo deben realizarse como se muestra en la Fig. 6.

Operación del servicio, es la última etapa del ciclo de vida y es crítica debido a que de ella depende la percepción del usuario para con el servicio; durante esta etapa se coordinan e implementan todas las actividades necesarias para la prestación de los servicios acordados con los niveles de calidad requeridos. Esta etapa es la encargada de dar soporte a los usuarios mediante la gestión de la infraestructura tecnológica y de proveer el servicio en las condiciones deseadas.

$\mathrm{Su}$ objetivo es cumplir con los lineamientos planteados y desarrollados a lo largo de las demás etapas del ciclo de vida; aportando resultados e indicadores a partir de los cuales se 
Tabla 5

Actividades a desarrollar en Operación del Servicio, Proceso ITIL.

\begin{tabular}{|c|c|c|}
\hline $\begin{array}{c}\text { Operación del } \\
\text { servicio }\end{array}$ & & Actividades \\
\hline \multirow[t]{3}{*}{ Gestión Eventos } & 33 & $\begin{array}{l}\text { Reporte por parte de los usuarios del } \\
\text { funcionamiento de la plataforma. }\end{array}$ \\
\hline & 34 & Reporte de incidencias. \\
\hline & 35 & $\begin{array}{l}\text { Diseño de formatos para la gestión de eventos } \\
\text { que se convirtieron en incidentes a cargo de la } \\
\text { Mesa de Ayuda. }\end{array}$ \\
\hline \multirow[t]{2}{*}{$\begin{array}{l}\text { Gestión de } \\
\text { Incidentes }\end{array}$} & 36 & $\begin{array}{l}\text { Las líneas de trabajo a nivel virtual deben ser } \\
\text { soportadas por Cloud Computing u otro } \\
\text { mecanismo y un servidor alterno de la } \\
\text { Universidad en caso de fallas. }\end{array}$ \\
\hline & 37 & $\begin{array}{l}\text { La mesa de ayuda es la encargada de brindar } \\
\text { soporte de incidencias al usuario. }\end{array}$ \\
\hline $\begin{array}{l}\text { Cumplimiento de } \\
\text { requerimientos }\end{array}$ & 38 & $\begin{array}{l}\text { Realizar pruebas periódicas de conectividad de } \\
\text { los servidores, atender solicitudes de } \\
\text { mejoramiento. }\end{array}$ \\
\hline \multirow{2}{*}{$\begin{array}{l}\text { Gestión de } \\
\text { problemas }\end{array}$} & 39 & $\begin{array}{l}\text { Establecer indicadores de rendimiento del } \\
\text { servicio. }\end{array}$ \\
\hline & 40 & $\begin{array}{l}\text { Monitorear el servicio en búsqueda de } \\
\text { incidencias y sus causas. }\end{array}$ \\
\hline \multirow{5}{*}{$\begin{array}{l}\text { Gestión de acceso } \\
\text { a los servicios }\end{array}$} & 41 & $\begin{array}{l}\text { Realizar control y seguimiento sobre cada } \\
\text { incidente generando reportes que permitan } \\
\text { trazabilidad. }\end{array}$ \\
\hline & 42 & $\begin{array}{l}\text { Disponer de recursos de consulta libre para los } \\
\text { usuarios con contenidos pertinentes como } \\
\text { cronogramas, materiales de apoyo y procesos } \\
\text { de interacción. }\end{array}$ \\
\hline & 43 & $\begin{array}{l}\text { Asegurar capacidad de acceso a los diferentes } \\
\text { recursos del curso en cualquier momento } \\
\text { teniendo en cuenta configuraciones de } \\
\text { visualización, funcionalidad de las } \\
\text { herramientas y que se cuente con acceso } \\
\text { independientemente del tipo de software a usar. }\end{array}$ \\
\hline & 44 & $\begin{array}{l}\text { Gestionar el acceso a software según criterio } \\
\text { para todos los usuarios en cualquier escenario. }\end{array}$ \\
\hline & 45 & $\begin{array}{l}\text { Garantizar los servicios de aulas virtuales, } \\
\text { video-conferencia, Webminars, Weblogs, } \\
\text { Bolsa de Empleo, Ecommerce y sistema de } \\
\text { asistencia en línea. }\end{array}$ \\
\hline
\end{tabular}

Fuente: Elaboración Propia.

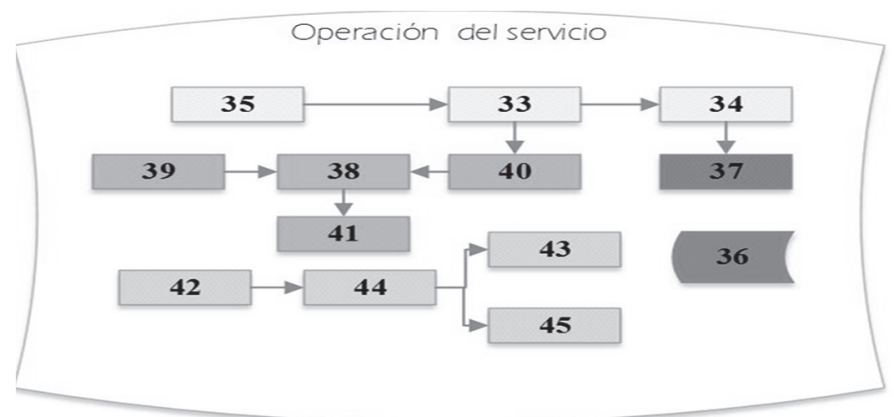

Figura 7. Diagrama de Flujo Operación del Servicio.

Fuente: Elaboración Propia

toman decisiones y se establece un nuevo punto de partida impulsado por el Mejoramiento continuo y gestionado desde el inicio del ciclo de vida por la etapa de Estrategia del servicio. Las actividades que aparecen en la Tabla 5, son vitales para cumplir con el objetivo de esta etapa.

El diagrama de flujo de la Fig. 7, evidencia las relaciones presentes para cada una de las actividades planteadas.

\section{Conclusión}

El modelo realizado demuestra que las prácticas que componen ITIL se pueden adaptar a los requerimientos de los programas de educación en metodología virtual, facilitando la gestión en la prestación de este tipo de servicios. La experiencia adquirida a través de la Maestría en Telecomunicaciones móviles de la Universidad Distrital permitió comprender la importancia de cada componente estudiado y establecer objetivos, funciones y actividades a desarrollar, formando así la base de un proceso de planeación estratégica al cual le hace falta designar personal que trabaje en pro de los objetivos y ejecute las actividades planteadas por MGEV.

Los casos de éxito en la implementación de prácticas ITIL estudiados previamente demuestran el valor añadido que brindan a un servicio, y aplicar un modelo basado en estas prácticas que busca satisfacer requerimientos pedagógicos, administrativos, comunicativos y tecnológicos, en un sistema de interacción directa e inmediata usuario - servicio, en donde los tiempos de respuesta a incidentes y peticiones se convierten en los indicadores que miden la satisfacción de los clientes, convierte a MGEV en una excelente opción para la consecución de los objetivos de un sistema con estas características.

\subsection{Trabajo futuro}

Se espera consolidar este modelo como base de un programa académico con metodología virtual exitoso, para ello es necesario realizar el despliegue respectivo de los procesos planteados por MGEV, hacer seguimiento a cada uno y establecer indicadores de rendimiento durante la prestación del servicio y se requiere detectar oportunidades de mejora sobre el modelo e implementar en éste los procesos restantes de la librería ITIL con el fin de completar el ciclo de vida en su totalidad

\section{Bibliografía}

[1] Bayona, S., Evangelista, J. and Uquiche, D., Método para Seleccionar software de gestión de cambios y gestión de incidencias de ITIL, Inf. Syst. Technol. (CISTI), 2015 10th iber. conf., pp. 1-6, 2015. DOI: 10.1109/CISTI.2015.7170399

[2] Area-Moreira, M. Hablemos más de métodos de enseñanza y menos de máquinas digitales: Los proyectos de trabajo a través de la WWW, Coop. Educ. del MCEP, 79, pp. 26-32, 2005.

[3] García-Aretio, L., Historia de la educación a distancia, Rev. Iberoam. Educ. a Distancia, 2(1), pp. 8-27, 2001. DOI: 10.5944/ried.2.1.2084

[4] Durán, R., Estay-Niculcar, C. y Álvarez, H., Adopción de buenas prácticas en la educación virtual en la educación superior, Aula Abierta, 43(2), pp. 77-86, 2015. DOI: 10.1016/j.aula.2015.01.001

[5] Nagles-Garcia, N. y Yong-Castillo, E., Tendencias en la educación virtual., en: Seminario de modelos innovadores en las aulas: Aprender en la sociedad del conocimiento, escuelas y tecnologías, 2015, pp. 1-19.

[6] García, A., Fundamento y componentes de la educación a distancia, Univ. Nac. Educ. a Distancia, pp. 28-39, 2006.

[7] de Terán-Martín, A.G. y Oltra-Badenes, R., Desarrollo del proceso de gestión de capacidad ITIL en una compañía de Outsourcing de TI, 3 c TIC Cuad. Desarro. Apl. a las TIC, 4(1), pp. 43-56, 2015.

[8] Ahmad, N. and Shamsudin, Z.M., Systematic approach to successful implementation of ITIL, Procedia Comput. Sci., 17, pp. 237-244, 2013. DOI: $10.1016 /$ j.procs.2013.05.032 
[9] Nieves, M. and Iqbal, M., Introdution, in ITIL Service Strategy, 2011th ed. London, England: TSO (The Stationery Office), 2011, pp. 3-4.

[10] Wang, H., Sun, S., Huang, Y. and Cheng, S., An ITIL-based IT service management model for garment enterprises, Proc. Int. Conf. Inf. Manag. Innov. Manag. Ind. Eng. ICIII 2008, 2, pp. 47-51, 2008. DOI: 10.1109/ICIII.2008.258

[11] Latrache, A., Nfaoui, E.L.H. and Boumhidi, J., Multi agent based incident management system according to ITIL, Intell. Syst. Comput. Vis., pp. 1-7, 2015. DOI: 10.1109/ISACV.2015.7105552

[12] Zhang, S., Wang, D., Zhigang, P. and Zong, Y., Organization ITIL process integration based on web services, 2009 WRI World Congr. Softw. Eng., 1, pp. 412-416, 2009. DOI: 10.1109/WCSE.2009.393

[13] Tituaña-Davila, G. y Yacchirema-Vargas, D., Modelo de gestion de TI para la educación virtual en el Sistema Universitario Ecuatoriano, Escuela Politecnica Nacional, 2009.

[14] Lagua, A.C., Reingeniería de procesos en la dirección de tecnología de información y comunicación DITIC-UTA en base a la biblioteca ITIL, Universidad Técnica de Ambato, 2015.

[15] Conza, A. y Medrano, L., Análisis de riesgos informáticos y elaboración de un plan de continuidad para la unidad de educación virtual CEC-EPN, Escuela Politecnica Nacional, 2015. DOI: 10.1017/CBO9781107415324.004

[16] PlanEsTIC- UD, Lineamientos para la educación en metodología virtual 2015, Primera Ed. Bogota: Policromía Digital S.A.S, 2015.

J.I. Palacios-Osma, recibió el título de Psicólogo en 1987 de la Universidad Santo Tomas de Aquino, el título de Sp. en Administración Hospitalaria en 1993 de la Escuela de Administración de Negocios - EAN, título de MSc. en Elearning en Redes Sociales en 2016 de la Universidad Internacional de la Rioja en España y Diplomado de Estudios Avanzados en Administración y Dirección de Empresas de la Universidad Pablo de Olavide en España. Docente universitario desde el año 1996 tanto de pregrado como postgrado en la Universidad Distrital Francisco José de Caldas en la Facultad de Ingeniería en asignatura de Investigación, Administración Informática, TIC y Empresas, integrante del Comité de PlanEsTIC de la Universidad desde 2010, integrante del Grupo de Investigación en Comercio Electrónico en Colombia GICOECOL. Actualmente es Coordinador del Proyecto Curricular de la Maestría en Telecomunicaciones Móviles, en metodología virtual de la Facultad de Ingeniería de la Universidad Distrital Francisco José de Caldas Bogotá, Colombia.

ORCID: 0000-0003-4701-4373

J.L. Rodríguez-Guzmán, estudiante de décimo semestre de Ingeniería Industrial de la Universidad Distrital Francisco José de Caldas, Bogotá, Colombia. Recibirá el título de Ingeniero Industrial en 2016. Sus intereses de investigación están orientados a: Nuevos sistemas de aprendizaje, E-learning, Optimización de Procesos y Simulación de procesos

ORCID: 0000-0002-0369-1500

C.X. García-Ramírez, estudiante de décimo semestre de Ingeniería Industrial de la Universidad Distrital Francisco José de Caldas, Bogotá, Colombia. Recibirá el título de Ingeniero Industrial en 2016. Sus intereses de investigación están orientados a tecnologías de la información, optimización de procesos y métodos de enseñanza.

ORCID: 0000-0002-0460-7286 